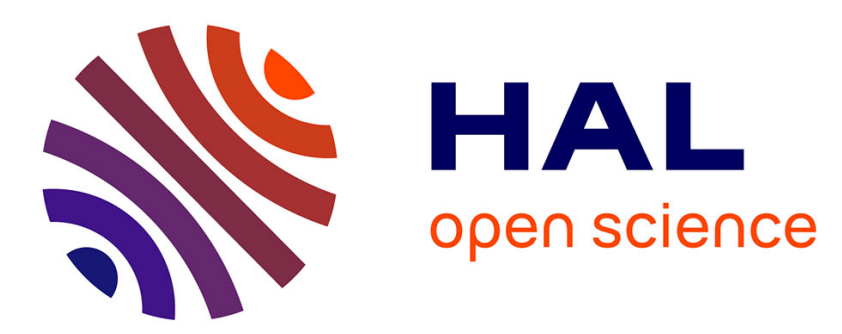

\title{
ISAR Image formation with a combined Empirical Mode Decomposition and Time-Frequency Representation
}

Ahmed Hadj Bay Ahmed, Jean-Christophe Cexus, Abdelmalek Toumi

\section{To cite this version:}

Ahmed Hadj Bay Ahmed, Jean-Christophe Cexus, Abdelmalek Toumi. ISAR Image formation with a combined Empirical Mode Decomposition and Time-Frequency Representation. EUSIPCO, Aug 2015, Nice, France. pp.1366-1370. hal-01241477

HAL Id: hal-01241477

https://hal-ensta-bretagne.archives-ouvertes.fr/hal-01241477

Submitted on 23 Feb 2016

HAL is a multi-disciplinary open access archive for the deposit and dissemination of scientific research documents, whether they are published or not. The documents may come from teaching and research institutions in France or abroad, or from public or private research centers.
L'archive ouverte pluridisciplinaire HAL, est destinée au dépôt et à la diffusion de documents scientifiques de niveau recherche, publiés ou non, émanant des établissements d'enseignement et de recherche français ou étrangers, des laboratoires publics ou privés. 


\title{
ISAR IMAGE FORMATION WITH A COMBINED EMPIRICAL MODE DECOMPOSITION AND TIME FREQUENCY REPRESENTATION
}

\author{
Bay Ahmed Hadj Ahmed ${ }^{* \dagger}$, Jean-Christophe Cexus*, Abdelmalek Toumi* \\ ${ }^{*}$ Lab-STICC, UMR CNRS 6285, \\ ENSTA Bretagne \\ 2, rue François Verny, \\ 29806 Brest Cedex 9, France. \\ email: $\{$ cexusje, toumiab\}@ensta-bretagne.fr \\ $\dagger$ IRENav, EA3634, \\ Ecole Navale \\ BCRM Brest, CC 600, \\ 29240 Brest Cedex 9, France. \\ email: hadj_ahmed.bay_ahmed@ecole-navale.fr
}

\begin{abstract}
In this paper, a method for Inverse Synthetic Aperture Radar (ISAR) image formation based on the use of the Complex Empirical Mode Decomposition (CEMD) is proposed. The CEMD [1] which based on the Empirical Mode Decomposition (EMD) is used in conjunction with a Time-Frequency Representation (TFR) to estimate a 3-D time-range-Doppler Cubic image, which we can use to effectively extract a sequence of ISAR 2-D range-Doppler images. The potential of the proposed method to construct ISAR image is illustrated by simulations results performed on synthetic data and compared to 2-D Fourier Transform and TFR methods. The simulation results indicate that this method can provide ISAR images with a good resolution. These results demonstrate the potential application of the proposed method for ISAR image formation.
\end{abstract}

Index Terms - Inverse Synthetic Aperture Radar, Image formation, Complex Empirical Mode Decomposition, TimeFrequency Representation.

\section{INTRODUCTION}

Nowadays in signal processing, spectrum analysis plays a key role to characterize and understand many phenomena and especially for Radar systems such as the Inverse Synthetic Aperture Radar (ISAR). However, for a non-stationary signal, the frequency components can appear or disappear. To deal with these temporal evolutions, a Time-Frequency Analysis must be considered $[2,3]$. The reconstruction process of ISAR image exploit the target's motions. Thus, ISAR images are usually obtained by the range-Doppler algorithm based on the 2-D Fourier Transform to convert the data in the spatial frequency domain to reflectivity information in the spatial domain. However, because of the target maneuvering, the Doppler spectrum becomes time-varying and the image is blurred. Instead of the Fourier Transform, TFR techniques can be adopted to improve the resolution of ISAR images [3-5]. In recent years, a great deal of interest has been paid to transformations or processing that map the signal into its TFR [5-7].

Recently, Empirical Mode Decomposition (EMD) has been introduced by Huang et al. [8] for analyzing data from nonstationary and nonlinear processes. It is a new local and fully data-driven method for the multiscale analysis of nonlinear and non-stationary real-world signals. Hence, the analysis is self-adaptive in contrast to the traditional methods where the basis functions are fixed [9]. The EMD is based on the sequential extraction of energy associated with various intrinsic time scales of the signal, called Intrinsic Mode Functions (IMF). The EMD has received more attention in terms of interpretations [10,11], improvement [1, 12-14], TimeFrequency Analysis [15-17], denoising [18, 19], Speech enhancement [20], and Radar field [21-25] ....

In this present work, we investigate an approach based on CEMD and TFR, called CEMD-TFR, for ISAR image construction. The paper is organized as follows. In section 2, we briefly review the EMD algorithm of real-valued data, and describe several extensions methods to the complex domain in order to handle the complex ISAR raw data. Section 3 gives an overview of ISAR formation with Time-Frequency Representation and the method based on EMD-TFR. Some simulations and comments are proposed in section 4. Finally, the last section gives some conclusions and remarks.

\section{METHOD DESCRIPTION}

\subsection{EMD algorithm}

The EMD method decomposes a signal $x(t)$ into a finite set of oscillatory modes, called Intrinsic Mode Functions (IMF), through an iterative process called sifting algorithm [8]. The name IMF is adopted because it represents the fast to the slow oscillations in the signal. The IMF represent the natural oscillatory mode embedded in signal and work as the basis functions. Usually, an IMF can be both amplitude and frequency modulated (AM-FM). The essence of the EMD is to identify the IMF in different time scales, which can be defined locally 
by the time lapse between two extrema of an oscillatory mode or by the time lapse between two zero crossings of such mode. The EMD picks out the highest frequency oscillation that remains in the signal. Thus, locally, each IMF contains lower frequency oscillations than the one extracted just before. The EMD decomposes a signal into a sum of IMF that: (R1) have the same number of zeros crossings and extrema; and (R2) are symmetric with respect to the local mean. The procedure used for extraction of an IMF from signal $x(t)$ is outlined in algorithm :

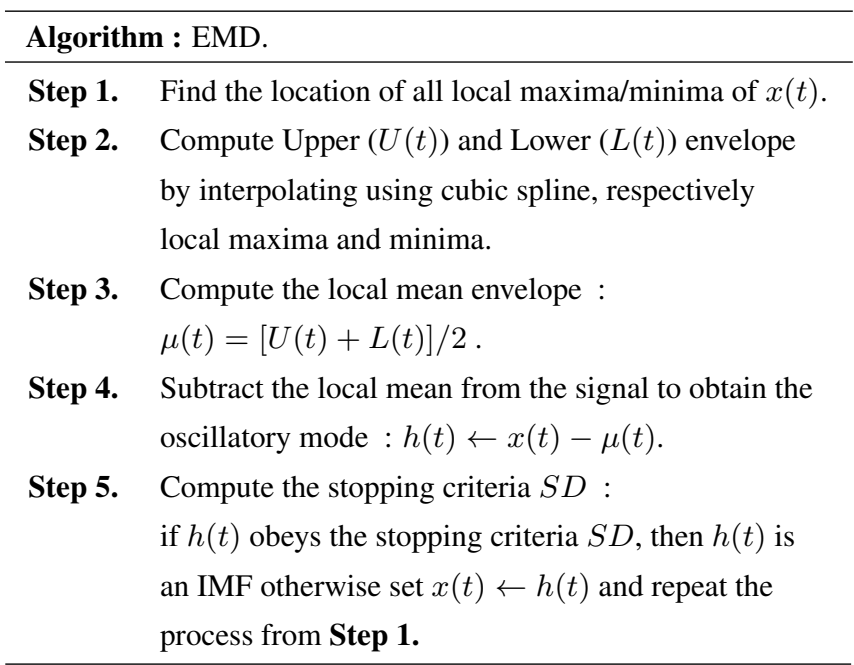

Once the first IMF is estimated, the same process is applied to the residual $x(t)-h(t)$ to extract the remaining IMFs. The sifting is also repeated several times in order to get $h(t)$ as to be a true IMF that fulfills the requirements (R1) and (R2). The result of the sifting procedure is that $x(t)$ will be decomposed into $I M F_{j}(t), j=1, \ldots N$ and residual $r_{N}(t)$ :

$$
x(t)=\sum_{j=1}^{N} I M F_{j}(t)+r_{N}(t) .
$$

The total sum of the IMFs matches the signal very well and therefore ensures completeness [8]. The sifting process has two effects: (a) eliminates riding waves and (b) smooth uneven amplitudes. To guarantee that the IMF components retain enough physical sens of both AM and FM informations, the stop criterion is originally based on the normalization squared difference between two successive sifting iterations [8]. It should be noted that there are other more robust criterion based upon the original definition of IMF $[10,11]$.

\subsection{Complex extension of EMD}

The original EMD can only be applied to real-valued time series, so it is necessary to extend the EMD to the complex domain. Despite original EMD becoming a standard for TimeFrequency analysis of nonlinear and non-stationary signals, its multivariate extensions, especially on complex signals (or images), are only emerging. Different methods have been proposed such as RIEMD [26], BEMD [12] and CEMD [1]. Besides these approaches, the extensions to trivariate and multivariate methods have also been developed and applied successfully $[13,14,25,27]$. It should be noted that the recent advances in applications of micro-Doppler effects have been presented in radar area based on EMD [22, 25, 28].

In [26], the authors proposed a method called Rotation Invariant Empirical Mode Decomposition (RIEMD), which differs from the original EMD in the way of getting the extrema and the envelopes. The extrema were thought as the locus where the first derivative of the angle changes its sign, and the maxima and minima were assumed to occur alternately. Then the cubic spline interpolation is performed directly in $\mathbb{C}$ to obtain the complex envelopes which are then averaged to obtain the local mean of signal. The other steps were same as the original EMD. This approach appears as a natural extension of real valued EMD.

In [12], Rilling et al. proposed another method termed Bivariate Empirical Mode Decomposition (BEMD) based on the idea of replacing the oscillation notion by a rotation notion. The complex signal was firstly projected to a certain directions to extract the maxima of the projection vector, and then connected these points forming the upper envelope. Repeat projecting the complex signal to different directions to obtain the envelopes in different directions. This approach effectively sifts rapidly rotating signal components from the slowly rotating ones and uses the same complex cubic spline interpolation scheme as RIEMD. It should be noted that BEDM algorithm calculates local mean envelope based on the extrema of both (real and imaginary) components of a complex signal and thus yielding more accurate estimates than RIEMD [26, 29].

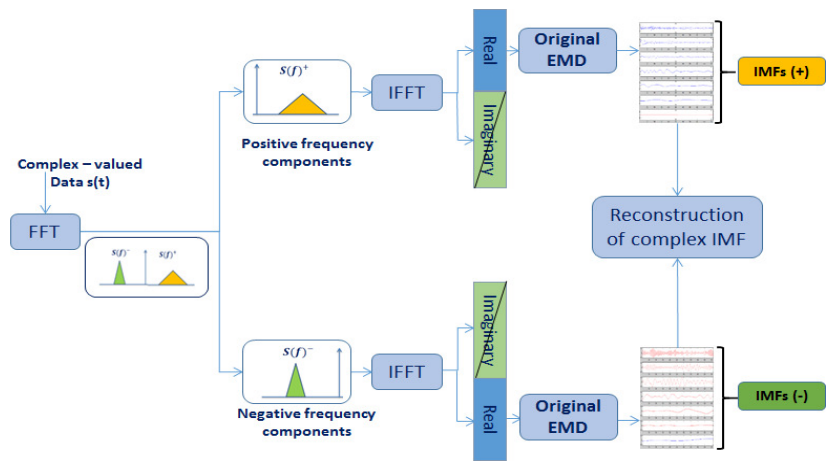

Fig. 1. Block diagram of CEMD algorithm.

In [1], the authors proposed a method named Complex Empirical Mode Decomposition (CEMD). This approach uses the inherent relationship between the positive and negative frequency components of a complex signal and the Hilbert Transform. Indeed, a complex signal has a asymmetric spectrum and can be converted into a sum of two analytic signals by first separating the positive and negative frequency components of the spectrum and then converting back into the time domain (Figure 1). And subsequently, the original EMD is 
applied to the two extracted signals. The algorithm has rigorous mathematical background and it preserves the dyadic filter bank properties [1,29]. However, this method cannot guarantee an equal number of real and imaginary IMFs. In the present work, this method was used because it seems more applicable to ISAR image construction.

\section{TIME-FREQUENCY REPRESENTATION-BASED ISAR FORMATION}

The common radar imaging method is based on the 2-D Fourier Transform (FT) and assumes that the Doppler frequency shifts must remain constant $[3,6]$. If input complex data consists of $M$ time history series, each one having length of $N$, then it should be noted that the 2-D FT based image formation generates only one image frame from $M \times N$ data array as shown in Figure 4(a). However, when the Doppler spectrum is time-varying due to target's motion, ISAR image based on Fourier Transform becomes unclear and smeared. However, the joint Time-Frequency Transform can be used to enhance the ISAR images of moving targets $[3,4,6]$.

\subsection{ISAR imaging based on TFR}

To compute a clear ISAR image of maneuvering targets, a Time-Frequency Transforms is always desirable. Figure 2 shows the radar imaging system based on the TFR [6].

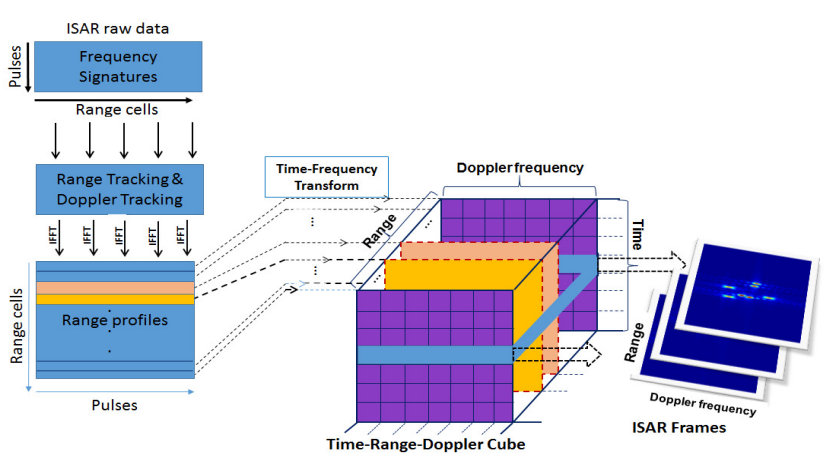

Fig. 2. Block diagram of Time-Frequency based ISAR image formation.

It should be noted that the Time-Frequency Transform based image formation takes the TFR for each time history series and generates an $N \times N$ time-Doppler representations. By combining the $M$ time-Doppler representations at $M$ range cells, we obtain a cube with $N \times M \times N$ time-range-Doppler values. There are also $N$ images frames availables and every one represents a full range-Doppler images at a particular time instant. Therefore, by replacing the Fourier Transform with the TFR, a 2-D range-Doppler Fourier image frame becomes a 3-D time-range-Doppler images cube. By sampling the image cube in time, a time sequence of 2-D range-Doppler images can be viewed. Each individual time-sampled frame from the cube provides not only a clear image with superior resolution but also time-varying properties from one time to another. Different methods have been proposed for ISAR imaging using TFRs such as Cohen class Time-Frequency distribution $[3,4,6]$, S-Transform [5,7] or Harmonic Wavelet [30].

\subsection{ISAR imaging based on EMD-TFR}

The approach of the present work combines the CEMD to TFR as Spectrogram, Wigner-Ville Distribution (WVD) or Smoothed Pseudo Wigner-Ville Distribution (SPWVD). The CEMD-TFR method can be divided into two parts. The first one deals with the separation of the signal into IMFs using the CEMD. In second part, the Time-Frequency Analysis is applied on the separated components (IMFs) using Spectrogram, WVD or SPWVD (Figure 3).

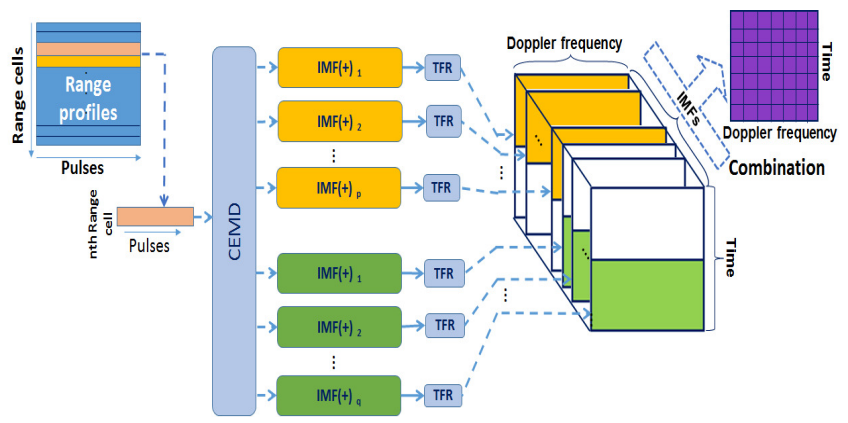

Fig. 3. Block diagram of CEMD-TFR algorithm based ISAR image formation.

Thus, the EMD is used as a multiband ltering to separate each components in the temporal domain before applying TFR. The intrinsic mode functions are used to construct a series of views by highlighting concentrations of energy in TimeFrequency domain. Therefore, we obtain a composite TFR based on a multiple views for each range profile signal. The advantage of such a method is that it can overcome interference in TFRs generated by the existence of multiple signal components. Once all TFRs associated with each IMF for a unique range cell are obtained, we compute a unique TFR (as a simple sum). Actually, the final result is an energy TFR at each range cell and along pulses, and time-Doppler spectrum is estimated. Then, by combining the time-Doppler spectrum at all range cells, we obtain a 3-D time-range-Doppler cubic image. Finally, the last step of classical image formation based on TFR is applied and a sequence of 2-D range Doppler images can be extracted.

\section{RESULTS}

We tested our approach on the simulated MIG25 dataset described in $[3,6]$. The simulated aircraft is composed of 120 point scatterers of equal reflectivity. The raw data contains 
512 recurrences of 64 range bins. For all simulations, standard motion compensation algorithm has been applied to the data. It should be noted that the representation of these figures is in decibel. The EMD-TFR applied to the cross-range dimension significantly improves image readability as it is presented in the Figure 6 compared to the conventional 2-D Fourier Transform (FT) (Figure 4(a)), Spectrogram (Figure 4(b)) and RTF (Figure 5). Figure 5(a) shows the ISAR image formation using WVD. The blurring effects due to the cross-term interference associated with the WVD are evident. To reduce the cross term interference, the SPWVD (Figure 5(b)) can be used to preserve TFR properties with slightly reduced time-frequency resolution and largely reduced crossterm interference. Comparing the EMD-WVD against the WVD, we see that in the figure 6(b) the components are well estimated and reduced cross-term interference with a good resolution. The simulation results show that this EMD-TFR can improve the constructed image compared with the Fourier Transform method. The EMD-TFR provides very interesting performance when the Time-Frequency energy is presented by the concentration and resolution of TFR along the individual component of the multi-components (non-stationary) signals.

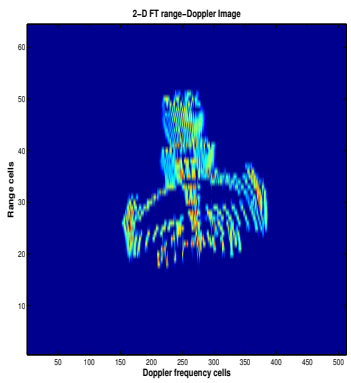

(a) Using 2-D FT.

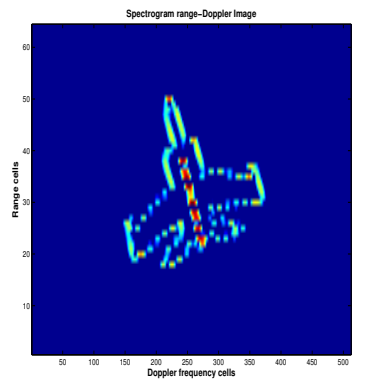

(b) Using Spectrogram (frame 10).
Fig. 4. Comparison of 2-D FT and Spectrogram based image.

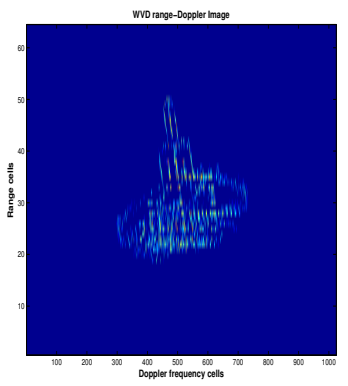

(a) Using WVD (frame 10).

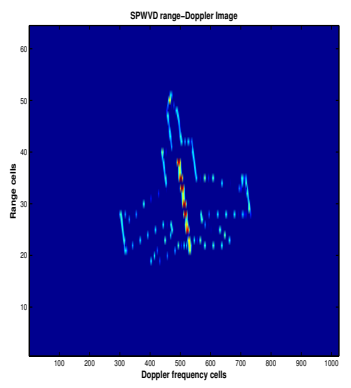

(b) Using SPWVD (frame 10)
Fig. 5. Comparison of Time-Frequency based image.

\section{CONCLUSIONS}

In this paper, a new method of ISAR image formation based on EMD and TFR is proposed in order to improve the ISAR

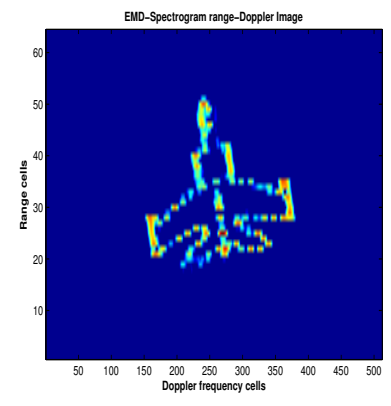

(a) Using EMD-Spectrogram (frame 10).

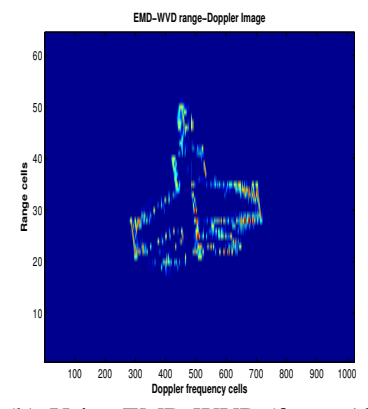

(b) Using EMD-WVD (frame 10)

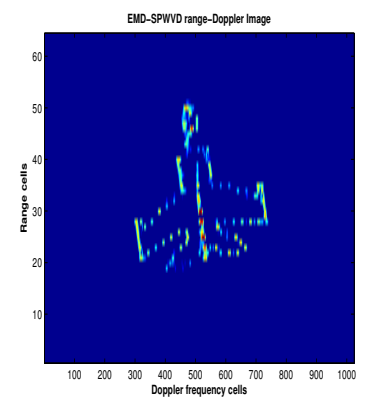

(c) Using EMD-SPWVD (frame 10).
Fig. 6. Comparison of EMD-TFR based image.

image resolution. The performance of the proposed method is compared with 2-D Fourier Transform method as well as conventional TFR methods (Spectrogram, WVD, SPWVD). The effectiveness of this approach has been veried with simulated data from a non-cooperative target. The obtained results show that the proposed approach is an effective and a promising imaging method for ISAR image formation. Nevertheless, this technique is empirical, so further theoretical explanation work is needed and a large class of data are necessary to confirm the obtained results. As future work, we plane to study the EMD-TFR in noisy environment and we intend to address the general decision-making process (classification problem and target identification). It seems also to be interesting to use others TFR such as the Huang-Hilbert Transform [8] or the Teager-Huang Transform [15].

\section{REFERENCES}

[1] T. Tanaka, and D.P. Mandic, "Complex Empirical Mode Decomposition," IEEE Signal Processing Letters, vol. 14(2), pp. 101-104, 2006.

[2] L. Cohen, "Time-frequency analysis: theory and applications," Prentice-Hall, Englewood Cliffs, NJ, USA, 299 pages, 1995.

[3] V.C. Chen, and H. Ling, "Time-Frequency Transforms for Radar Imaging and Signal Analysis," Artech House, Boston, 232 pages, 2002.

[4] V. Corretja, E. Grivel, et al., "Enhanced Cohen class Time-Frequency methods based on a structure tensor 
analysis: Applications to ISAR processing," Signal Processing, vol. 93(7), pp. 1813-1830, 2013.

[5] I. Djurovic, L. Stankovic, et al., "Time-Frequency Analysis for SAR and ISAR Imaging," GeoSpatial Visual Analytics, Springer, pp. 113-127, 2009.

[6] V.C. Chen, and M. Martorella, "Inverse Synthetic Aperture Radar Imaging; Principles, Algorithms and Applications," SciTech Publishing, 289 pages, 2014.

[7] L.J. Stankovic, T. Thayaparan, et al., "S-method in Radar Imaging," EUSIPCO'06, 5 pages, 2006.

[8] N.E. Huang, Z. Shen, et al., "The Empirical Mode Decomposition and the Hilbert spectrum for nonlinear and non-stationary time series analysis," Proceedings of the Royal Society, A, vol. 454, pp. 903-995, 1998.

[9] P. Flandrin, G. Rilling, and P. Goncalves, "Empirical Mode Decomposition as a Filter Bank," IEEE Signal Processing Letters, vol. 11(2), pp. 112-114, 2004.

[10] N.E. Huang, M.L.C Wu, et al., "A confidence limit for the Empirical Mode Decomposition and Hilbert spectral analysis," Proceedings of the Royal Society, A, vol. 459, pp. 2317-2345, 2003.

[11] G. Rilling, P. Flandrin, and P. Goncalves, "On Empirical Mode Decomposition and its algorithms," EURASIP workshop on nonlinear signal and image processing, NSIP-03, vol. 3, pp.8-11, 2003.

[12] G. Rilling, P. Flandrin, P. Goncalves, and J.M. Lilly, "Bivariate Empirical Mode Decomposition," IEEE Signal Processing Letters, vol. 14(12), pp. 936-939, 2007.

[13] N. Rehman, and D.P. Mandic, "Multivariate Empirical Mode Decomposition," Proceedings of the Royal Society, A, vol. 466, pp. 1291-1302, 2009.

[14] M.H. Yeh, "The complex bidimensional Empirical Mode Decomposition," Signal Processing, vol. 92(2), pp. 523-541, 2012.

[15] J.C. Cexus, and A.O. Boudraa, "Non-stationary signals analysis by Teager-Huang Transform (THT)," EUSIPCO'06, 5 pages, 2006.

[16] A. Bouchikhi, A.O. Boudraa, J.C. Cexus, and T. Chonavel, "Analysis of multicomponent LFM signals by Teager Huang-Hough Transform," Aerospace and Electronic Systems, IEEE Transactions on, vol. 50(2), pp. 1222-1233, 2014.

[17] N.J. Stevenson, M. Mesbah, and B. Boashash, "Multiple-view time-frequency distribution based on the Empirical Mode Decomposition," IET signal processing, vol. 4(4), pp. 447-456, 2010.

[18] A.O. Boudraa, and J.C. Cexus, "EMD-Based Signal Filtering," Instrumentation and Measurement, IEEE Transactions on, vol. 56(6), pp.2196-2202, 2007.
[19] P. Flandrin, P. Goncalves, and G. Rilling, "Detrending and denoising with Empirical Mode Decomposition," EUSIPCO'04,, 4 pages, 2004.

[20] K. Khaldi, A.O. Boudraa, A. Bouchikhi, and M.T. Alouane, "Speech enhancement via EMD," Journal on Advances in Signal Processing,, vol. 2008(1), 8 pages, 2008.

[21] H. Chunming, G. Huadong, W. Changlin, and F. Dian, "A novel method to reduce speckle in SAR images," International Journal Remote Sensing, vol. 23(23), pp. 5095-5101, 2002.

[22] L. Du, B. Wang, Y. Li, and H. Liu, "Robust classification scheme for airplane targets with low resolution radar based on EMD-CLEAN feature extraction method," IEEE Sensors Journal, vol. 13(12), pp. 46484662, 2013.

[23] B. Bjelica, M. Dakovic, et al.,"Complex Empirical Decomposition method in radar signal processing," Embedded Computing (MECO), 2012 Mediterranean Conference on, pp. 88-91, 2012

[24] Y. Yuani, M. Jin, et al., "Empiric and dynamic detection of the sea bottom topography from synthetic aperture radar image," Advances in Adaptive Data Analysis, vol. 1(2), pp. 243-263, 2009.

[25] J.H. Park, W. Y. Yang, et al., "Extended high resolution range profile-jet engine modulation analysis with signal eccentricity," Progress In Electromagnetics Research, vol. 142, pp. 505-521, 2013.

[26] B. Altaf, M. Umair, et al., "Rotation invariant complex Empirical Mode Decomposition," ICASSP07, vol. 3, pp. 1009-1012, 2007.

[27] N.U. Rehman, and D.P. Mandic, "Empirical mode decomposition for trivariate signals," IEEE Transactions on Signal Processing, vol. 58 (3), pp. 1059-1068,2010.]

[28] C. Cai, W. Liu, et al., "Radar micro-Doppler signature analysis with HHT,' IEEE Trans. Aerosp. Electron. Syst. vol. 46(2), pp. 929-938, 2010.

[29] D.P. Mandic, and V.S.L. Goh, "Complex Valued Nonlinear Adaptive Filters: Noncircularity, Widely Linear and Neural Models," John Wiley \& Sons, 344 pages, 2009.

[30] B.K. Kumar, B. Prabhakar, et al., "Target identification using harmonic wavelet based ISAR imaging," Journal on Applied Signal Processing, vol. 2006(1), pp. 1-13, 2006. 